\title{
LINC00978 promotes bladder cancer cell proliferation, migration and invasion by sponging miR-4288
}

\author{
WEI WANG ${ }^{1}$, ZHIHUI XU ${ }^{2}$, JIYE WANG $^{1}$ and RENZONG CHEN ${ }^{1}$ \\ ${ }^{1}$ Department of Urinary Surgery, Tiantai People's Hospital of Zhejiang Province, Taizhou, Zhejiang 317200; \\ ${ }^{2}$ Department of Urinary Surgery, Zhejiang Provincial People's Hospital, Hangzhou, Zhejiang 310014, P.R. China
}

Received March 30, 2018; Accepted November 9, 2018

DOI: $10.3892 / \mathrm{mmr} .2019 .10395$

\begin{abstract}
Long noncoding RNAs regulate the occurrence and progression of numerous types of cancer, including bladder cancer $(\mathrm{BCa})$. However, the role of long intergenic non-protein coding RNA 978 (LINC00978) in BCa remains unknown. In the present study, it was identified that the LINC00978 expression level was significantly increased in BCa tissues compared with adjacent normal tissues. By performing cell proliferation, colony formation, Transwell and Matrigel assays, it was demonstrated that LINC00978 knockdown significantly inhibited the proliferation, migration and invasion of $\mathrm{BCa}$ cells. Conversely, overexpression of LINC00978 resulted in opposite effects. Mechanistically, it was demonstrated that LINC00978 served as a competing endogenous RNA to sponge microRNA-4288 (miR-4288), and LINC00978 knockdown significantly increased the expression level of miR-4288 in $\mathrm{BCa}$ cells. Furthermore, it was demonstrated that the expression of LINC00978 was inversely correlated with the miR-4288 expression level in BCa tissues. Furthermore, by performing a rescue assay, it was identified that inhibition of miR-4288 was able to reverse the effects of LINC00978 knockdown on proliferation, migration and invasion of BCa cells. Collectively, the present study demonstrated that LINC00978 promoted $\mathrm{BCa}$ progression by sponging miR-4288, suggesting that LINC00978 may represent a potential therapeutic target for BCa treatment.
\end{abstract}

\section{Introduction}

Bladder cancer $(\mathrm{BCa})$ is the second most common cancer of the genitourinary tract (1), causing numerous cancer-associated mortalities (2). The incidence of $\mathrm{BCa}$ has increased over the past decades, and specific therapeutic strategies were

Correspondence to: Professor Wei Wang, Department of Urinary Surgery, Tiantai People's Hospital of Zhejiang Province, 335 Labor Road, Taizhou, Zhejiang 317200, P.R. China

E-mail: wangwei16000@163.com

Key words: long intergenic non-protein coding RNA 978, microRNA-4288, bladder cancer, proliferation, migration, invasion developed to treat $\mathrm{BCa}$, including endoscopic resection and radical cystectomy (3). However, due to recurrence, drug resistance and tumor metastasis, the outcomes of patients with $\mathrm{BCa}$ remain poor (4). Therefore, in order to identify better therapeutic targets and develop more effective therapeutic methods, understanding the mechanism underlying $\mathrm{BCa}$ progression is required.

The number of identified long noncoding RNAs (lncRNAs) has been increasing (5). IncRNAs are $>200$ nucleotides in length and do not possess protein-coding potential (6). Accumulating evidence suggests that lncRNAs serve important roles in numerous biological processes, including immunity, embryonic development and cancer (7-9). In humans, a previous study demonstrated that IncRNAs serve roles in cancer progression by regulating cell proliferation, migration, invasion and apoptosis (10). In BCa, numerous lncRNAs were demonstrated to be aberrantly expressed, and were associated with $\mathrm{BCa}$ progression. For example, lncRNA bladder cancer-associated transcript 2 promoted BCa metastasis (11), and lncRNA fibroblast growth factor receptor 3 antisense transcript 1 promoted BCa growth and metastasis (12). Additionally, IncRNA urothelial cancer associated 1 contributed to $\mathrm{BCa}$ progression and metastasis by modulating the microRNA (miR)-143/high mobility group box 1 signaling pathway (13).

Previous studies suggested that LINC00978 promoted the development of gastric cancer and breast cancer $(14,15)$. However, the role of LINC00978 in BCa has not been investigated. The present study identified that LINC00978 was upregulated in BCa tissues. Furthermore, knockdown of LINC00978 suppressed the proliferation, migration and invasion of $\mathrm{BCa}$ cells. Mechanistically, it was identified that LINC00978 served as a competing endogenous RNA (ceRNA) of miR-4288. The present study demonstrated that LINC00978 promoted $\mathrm{BCa}$ cell proliferation, migration and invasion by inhibiting miR-4288. Collectively, the present study suggested that the LINC00978/miR-4288 axis is an important regulator of $\mathrm{BCa}$ progression.

\section{Materials and methods}

Clinical specimens and cell lines. A total of $60 \mathrm{BCa}$ (46 males and 14 females; age range, 37-61 years old; median age, 51 years old) and adjacent normal tissue samples were obtained from The Tiantai People's Hospital of Zhejiang 
Province (Taizhou, China) between September 2014 and November 2016. Written informed consent was obtained from all enrolled patients. Patients treated by radiotherapy or chemotherapy before surgery were excluded. All other patients were included. The present study was approved by The Ethics Committee of Tiantai People's Hospital of Zhejiang Province (approval no. 2016020342). A total of four BCa cell lines (T24, J82, UMUC3 and 5637) and a normal bladder cell line (SV-HUC-1) were purchased from The Cell Bank of The Chinese Academy of Sciences (Shanghai, China). Cells were cultured in the Dulbecco's modified Eagle's medium (DMEM; Gibco; Thermo Fisher Scientific, Inc., Waltham, MA, USA) supplemented with $10 \%$ fetal bovine serum (FBS; Gibco; Thermo Fisher Scientific, Inc.) and maintained at $37^{\circ} \mathrm{C}$ in a cell incubator with a humidified atmosphere containing $5 \% \mathrm{CO}_{2}$.

Oligonucleotide and transfection. A small interfering RNA against LINC00978 (siLINC00978; 5'-GGACAGAGCAGA AGACAAA-3'), siRNA negative control (siNC; 5'-AAU UCUCCGAACGUGUCAC-3'), miR-4288 mimics (5'-UUG UCUGCUGAGUUUCC-3'), miR-4288 inhibitors (5'-GGA AACUCAGCAGACAA-3') and controls (5'-UCACAA CCUCCUAGAAAGAGUAGA-3') were purchased from GeneCopoeia, Inc. (Rockville, MD, USA). To overexpress LINC00978, its coding sequence was constructed into pcDNA3 vector (Invitrogen; Thermo Fisher Scientific, Inc.) to generate pcDNA3-LINC00978. The siRNAs (100 nM), mimics (100 $\mathrm{nM})$, inhibitor (100 nM) r, pcDNA3-LINC00978 $(1 \mu \mathrm{g})$ and their corresponding negative controls $(100 \mathrm{nM}$ or $1 \mu \mathrm{g})$ were transfected into T24 cells using Lipofectamine ${ }^{\circledR}$ 2000 transfection reagent (Invitrogen; Thermo Fisher Scientific, Inc.) according to the manufacturer's protocol. After $48 \mathrm{~h}$, the transfection efficiency was confirmed by reverse transcription-quantitative polymerase chain reaction (RT-qPCR) as described below.

Cell proliferation assay. Cell proliferation was measured by Cell Counting kit-8 (CCK-8; Dojindo Molecular Technologies, Inc., Kumamoto, Japan). A total of $5 \times 10^{3}$ cells were seeded in 96-well plates and incubated for 1, 2 and 3 days. Subsequently, $10 \mu \mathrm{l}$ CCK-8 reagent was added to the 96-well plates. Following $2 \mathrm{~h}$ incubation at $37^{\circ} \mathrm{C}$, the absorbance at $450 \mathrm{~nm}$ was measured to detect the number of viable cells using a SUNRISE microplate reader (Tecan Group, Ltd., Männedorf, Switzerland).

Colony formation assay. A total of $5 \times 10^{2} \mathrm{~T} 24$ cells transfected with siLINC00978 or siNC were seeded in 6-well plates. The culture medium was replaced every other day. Following incubation for 10 days, the colonies from three groups were fixed with ice-cold $100 \%$ methanol for $2 \mathrm{~h}$ at $4^{\circ} \mathrm{C}$ and stained with $0.1 \%$ crystal violet for $1 \mathrm{~h}$ at room temperature. Subsequently, the number of colonies was determined using a light microscope (magnification, x100; Nikon Corporation, Tokyo, Japan).

$R T$ and $R T-q P C R$. Total RNA was extracted from cultured cells using TRIzol ${ }^{\circledR}$ reagent (Invitrogen; Thermo Fisher Scientific, Inc.) according to the manufacturer's protocol and cDNA was synthesized from $1 \mu \mathrm{g}$ total RNA using a PrimerScript RT reagent kit (Takara Bio, Inc., Otsu, Japan). The RT conditions were: Incubation for $1 \mathrm{~h}$ at $42^{\circ} \mathrm{C}$ and inactivation at $70^{\circ} \mathrm{C}$ for 15 min. miRNA from total RNA was reverse transcribed using the PrimeScript miRNA cDNA synthesis kit (Takara Bio, Inc.). RT-qPCR was performed with the SYBR-Green Premix Ex Taq II (Takara Bio, Inc.) using the StepOnePlus RT PCR system (Applied Biosystems; Thermo Fisher Scientific, Inc.). The PCR thermocycling conditions were: $95^{\circ} \mathrm{C}$ for $30 \mathrm{sec}$, followed by 40 cycles of $95^{\circ} \mathrm{C}$ for $5 \mathrm{sec}$ and $60^{\circ} \mathrm{C}$ for 34 sec. U6 was used as the endogenous control for miRNA and LINC00978 expression analysis. The relative gene expression level was calculated using the $2^{-\Delta \Delta \mathrm{Cq}}$ method (16). The sequences of the primers were: LINC00978 (Forward, 5'-TAG GAGACACAGGCAGAGCC-3' and reverse, 5'-CTGACT AGAGCTTGTCTCA-3'), miR-4288 (Forward, 5'-AACGAG ACGACGACAGAC-3' and reverse, 5'-TTGTCTGCTGAGTT TCC-3') and U6 (Forward, 5'-AACGAGACGACGACAGAC-3' and reverse, 5'-GCAAATTCGTGAAGCGTTCCATA-3').

Transwell and Matrigel assays. For Transwell migration, following transfection of siLINC00978 or siNC, $5 \times 10^{3} \mathrm{BCa}$ cells were plated in the upper chamber (Corning, Inc., Corning, NY, USA) containing $200 \mu \mathrm{l}$ serum-free DMEM. A total of $600 \mu \mathrm{l}$ DMEM containing 10\% FBS was added to the lower chamber. Following an incubation of $24 \mathrm{~h}$, the cells migrated into the lower chamber were fixed with $100 \%$ methanol at $4^{\circ} \mathrm{C}$ and stained with $1 \%$ crystal violet for $30 \mathrm{~min}$ at room temperature. Cells were imaged using a light microscope at magnification, x100. For the Matrigel assay, the upper chamber was pre-coated with Matrigel (1:3; Becton, Dickinson and Company, Franklin, Lakes, NJ, USA). The invasion assay was performed following the protocol used for the migration assay.

Luciferase reporter assay. The potential binding site for miR-4288 in LINC00978 were predicted by the miRDB tool (http://mirdb.org/miRDB/index.html). Then the sequence containing the wild-type or mutant binding site was constructed into pMIR-Report vector (Promega Corporation, Madison, WI, USA). For luciferase reporter assay, miR-4288 mimics and wild-type or mutant LINC00978 reporter were co-transfected into the cells using Lipofectamine ${ }^{\circledR} 2000$ (Invitrogen; Thermo Fisher Scientific, Inc.). Following transfection, cells were incubated for $24 \mathrm{~h}$, and luciferase activity was determined with a luciferase assay system kit (Promega Corporation). Renilla luciferase activity was used for normalization.

Statistical analysis. Statistical analysis was conducted using SPSS 20.0 (IBM Corp., Armonk, NY, USA). Data from three independent experiments were expressed as mean \pm standard deviation. Significant differences were calculated using Student's t-test or one-way analysis of variance followed by Tukey's post hoc test. Pearson's correlation coefficient analysis was used to determine correlation. $\mathrm{P}<0.05$ was considered to indicate a statistically significant difference.

\section{Results}

LINC00978 is upregulated in BCa tissues. To investigate the role of LINC00978 in $\mathrm{BCa}$, the expression level of LINC00978 was analyzed by RT-qPCR. LINC00978 was significantly upregulated in $\mathrm{BCa}$ tissues compared with 

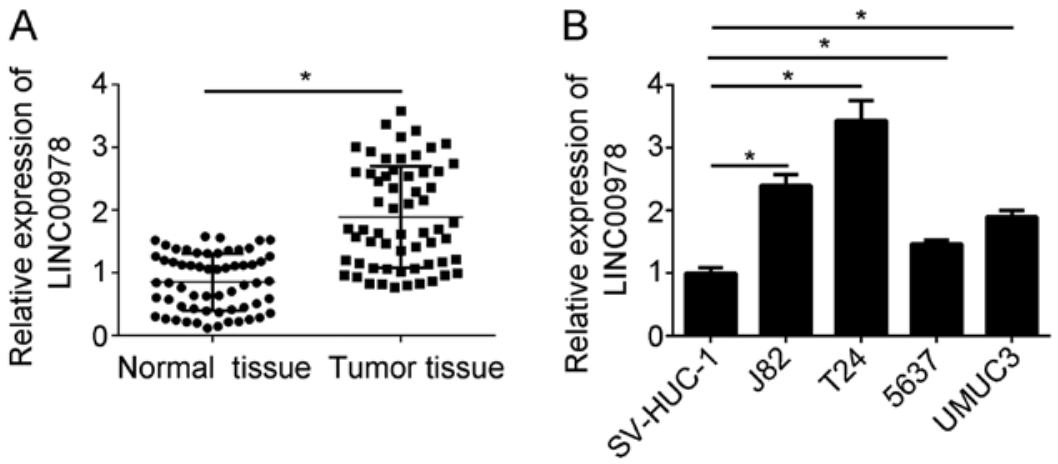

Figure 1. LINC00978 is upregulated in BCa tissues and cells. (A) Relative expression of LINC00978 in 60 samples of BCa tissues and respective adjacent normal tissues detected by RT-qPCR. (B) Relative expression of LINC00978 in BCa cell lines detected by RT-qPCR. "P<0.05. LINC00978, long intergenic non-protein coding RNA 978; BCa, bladder cancer; RT-qPCR, reverse transcription-quantitative polymerase chain reaction.
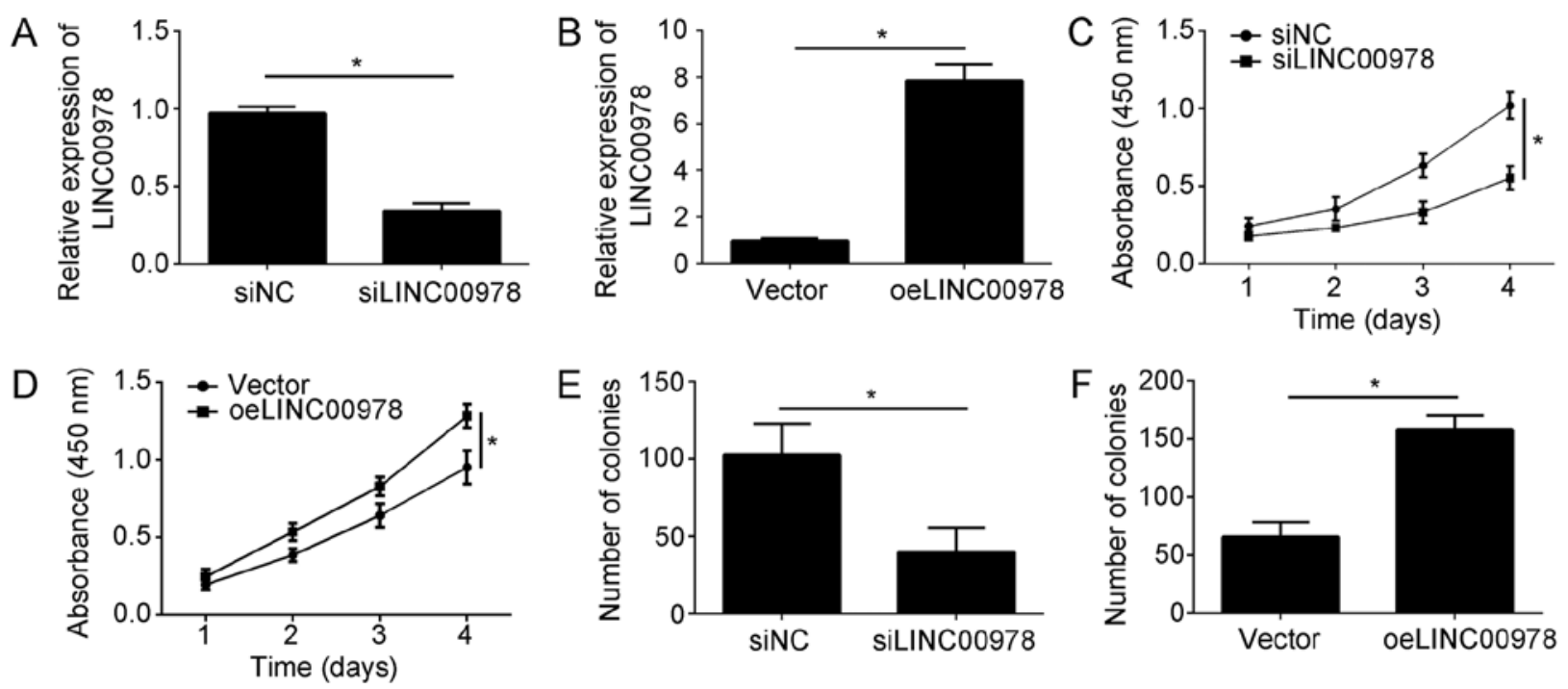

Figure 2. LINC00978 promotes bladder cancer cell proliferation. (A) Relative expression of LINC00978 in T24 cells transfected with siLINC00978 or siNC. (B) Relative expression of LINC00978 in T24 cells transfected with oeLINC00978 or an empty vector. Cell Counting kit-8 assay was used to measure the proliferation of cells with (C) downregulated and (D) upregulated expression of LINC00978. Colony formation assay was used to measure the proliferation of cells with (E) downregulated and (F) upregulated expression of LINC00978. "P<0.05. LINC00978, long intergenic non-protein coding RNA 978; si, small interfering; $\mathrm{NC}$, negative control; oe, overexpressed.

adjacent normal tissues (Fig. 1A). Furthermore, the expression level of LINC00978 was examined in BCa cell lines. The results suggested that LINC00978 was significantly upregulated in BCa cell lines, including J82, T24, 5637 and UMUC3 cells, compared with SV-HUC-1 cells (Fig. 1B). The present results suggested that LINC00978 may be involved in BCa progression.

LINC00978 promotes BCa cell proliferation. To investigate the function of LINC00978 in BCa, a knockdown of LINC00978 was performed by transfecting cells with siLINC00978. Additionally, overexpression of LINC00978 was conducted by transfecting T24 cells with a plasmid expressing LINC00978. RT-qPCR analysis demonstrated that the LINC00978 expression level was significantly decreased upon knockdown and upregulated following overexpression (Fig. 2A and B). Subsequently, a CCK-8 assay was conducted. LINC00978 knockdown significantly inhibited the proliferation of T24 cells at 4 days (Fig. 2C). Conversely, LINC00978 overexpression promoted cell proliferation at 4 days (Fig. 2D).
To test the effect of LINC00978 on BCa cell proliferation, a colony formation assay was additionally conducted. The results suggested that LINC00978 knockdown led to a significant decrease in the number of colonies (Fig. 2E), whereas, LINC00978 overexpression caused an increased number of colonies (Fig. 2F). Collectively, the present data suggested that LINC00978 promoted the proliferation of $\mathrm{BCa}$ cells.

LINC00978 promotes BCa cell migration and invasion. Malignancies are frequently associated with tumor metastasis. To test whether LINC00978 had an effect on BCa metastasis, a Transwell assay was conducted, using T24 cells. LINC00978 knockdown significantly inhibited migration (Fig. 3A), whereas, overexpression of LINC00978 caused the opposite effect (Fig. 3B). Similarly, LINC00978 knockdown decreased the number of invasive cells (Fig. 3C), and overexpression of LINC00978 promoted invasion (Fig. 3D). The present results suggested that LINC00978 contributed to the metastasis of BCa. 
A

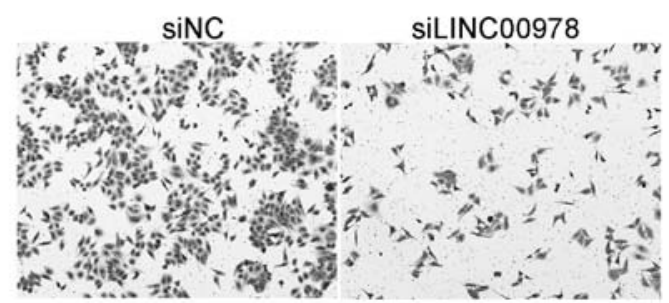

B

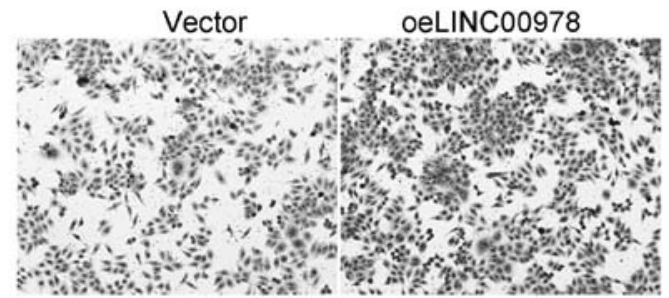

C

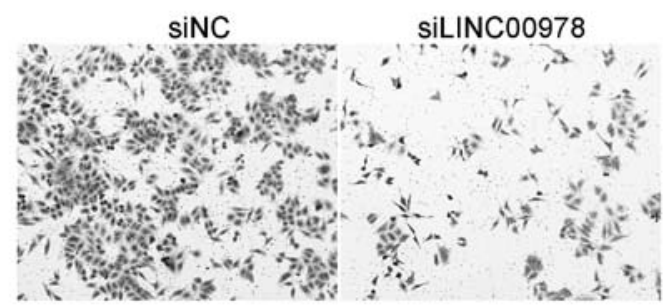

D

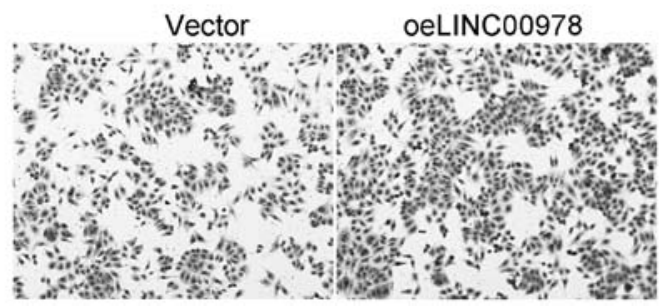

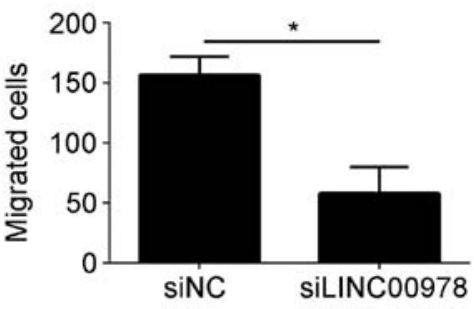
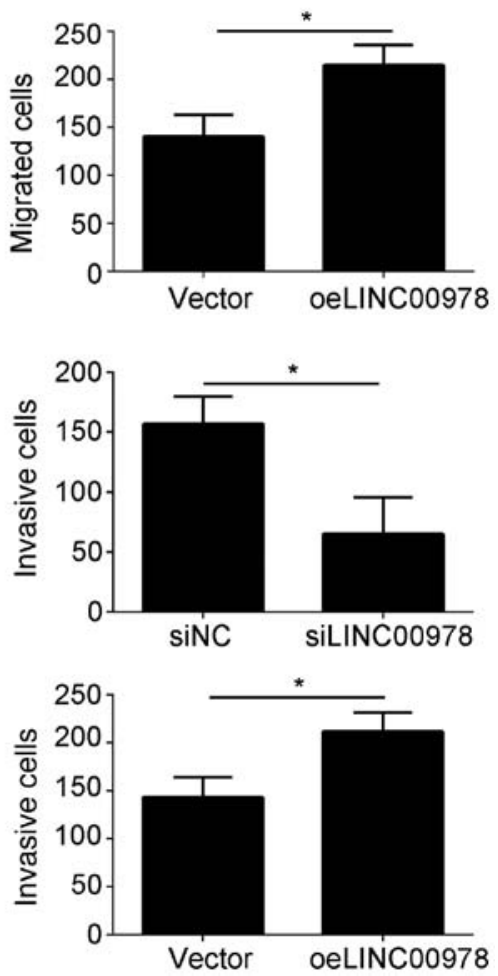

Figure 3. LINC00978 promotes bladder cancer cell migration and invasion. Transwell migration assay was used to analyze the migrating potential of T24 cells with (A) downregulated and (B) upregulated expression of LINC00978 (magnification, x100). Matrigel invasion assay was used to examine the invasive ability of T24 cells with (C) downregulated and (D) upregulated expression of LINC00978 (magnification, x100). "P<0.05. LINC00978, long intergenic non-protein coding RNA 978; si, small interfering; NC, negative control; oe, overexpressed.

LINC00978 sponges miR-4288 in BCa cells. To investigate the mechanism underlying LINC00978 function in BCa, a bioinformatics analysis was performed. It was identified that LINC00978 may serve as a ceRNA of miR-4288. In total, four predicted binding sites of miR-4288 were identified in LINC00978 (Fig. 4A). To test this prediction, a luciferase reporter assay was performed, using wild-type (WT) or mutant binding sites in a reporter plasmid. The results demonstrated that overexpression of $\mathrm{miR}-4288$ increased the expression level of miR-4288 (Fig. 4B), and suppressed the luciferase activity of WT-LINC00978 (Fig. 4C). Notably, mutation of the predicted binding sites abrogated this interaction (Fig. 4C). Furthermore, using RT-qPCR, it was identified that miR-4288 overexpression significantly decreased the expression level of LINC00978 in T24 cells (Fig. 4D). Notably, LINC00978 knockdown increased the expression level of miR-4288 in T24 cells (Fig. 4E). Furthermore, the expression level of LINC00978 was inversely correlated with miR-4288 expression level in BCa tissues (Fig. 4F). Collectively, the present data suggested that LINC00978 binds to miR-4288, inhibiting its expression in $\mathrm{BCa}$ cells.

Inhibition of miR-4288 reverses the effects of LINC00978 knockdown on BCa cells. As the present study demonstrated that LINC00978 interacted with miR-4288, it was examined whether miR-4288 was involved in regulating the function of LINC00978 in BCa cells. miR-4288 was inhibited in LINC00978-silenced T24 cells using miR-4288 inhibitors (Fig. 5A). RT-qPCR analysis demonstrated that the miR-4288 expression level was restored in LINC00978-silenced T24 cells with miR-4288 inhibitors (Fig. 5A). Subsequently, CCK-8, Transwell and Matrigel assays were conducted. LINC00978 knockdown inhibited the proliferation, migration and invasion of T24 cells, whereas, inhibition of miR-4288 was able to abrogate the effects of LINC00978 knockdown (Fig. 5B-D). Collectively, the present data demonstrated that LINC00978 promoted the proliferation, migration and invasion of BCa cells by inhibiting miR-4288.

\section{Discussion}

$\mathrm{BCa}$ is one of the most common types of cancer, leading to numerous mortalities every year (17). $\mathrm{BCa}$ is divided into non-muscle-invasive $\mathrm{BCa}(\sim 80 \%)$ and muscle-invasive $\mathrm{BCa}$ $(\sim 20 \%)$, based on the severity of tumor infiltration into the musculature of the bladder (18). BCa is a public health problem worldwide (2). However, the underlying mechanism of $\mathrm{BCa}$ 

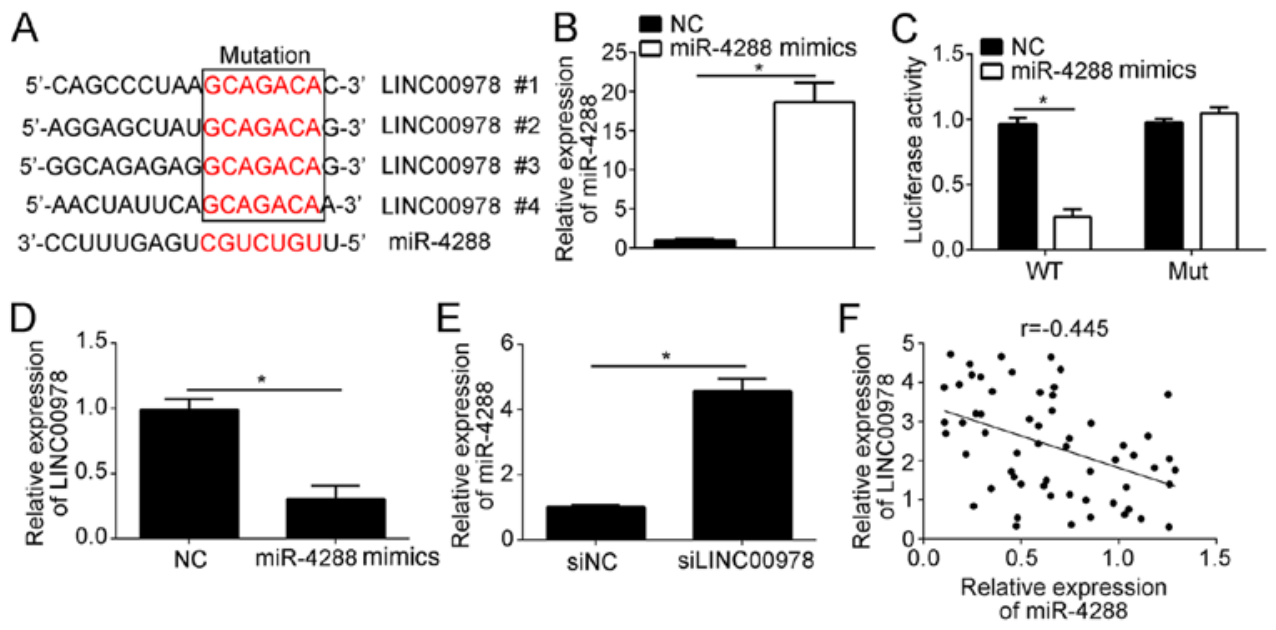

Figure 4. LINC00978 sponges miR-4288 in BCa cells. (A) Schematic of miR-4288 target sites in LINC00978. (B) Relative expression level of miR-4288 in T24 cells following transfection with miR-4288 mimics or NC. (C) Luciferase reporter assay was conducted to determine the interaction between LINC00978 and miR-4288 in T24 cells. (D) Overexpression of miR-4288 decreased the expression level of LINC00978 in T24 cells. (E) Knockdown of LINC00978 increased the expression level of miR-4288 in T24 cells. (F) Correlation between LINC00978 and miR-4288 expression levels in BCa tissues by reverse transcription-quantitative polymerase chain reaction. ${ }^{*} \mathrm{P}<0.05$. LINC00978, long intergenic non-protein coding RNA 978; miR-4288, microRNA-4288; WT, wild-type; Mut, mutant; si, small interfering; NC, negative control; BCa, bladder cancer.

A

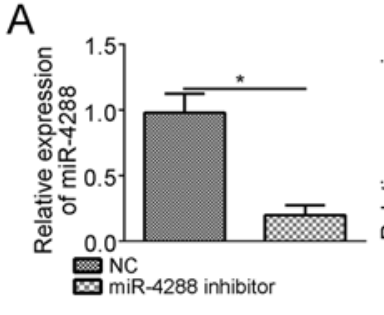

C

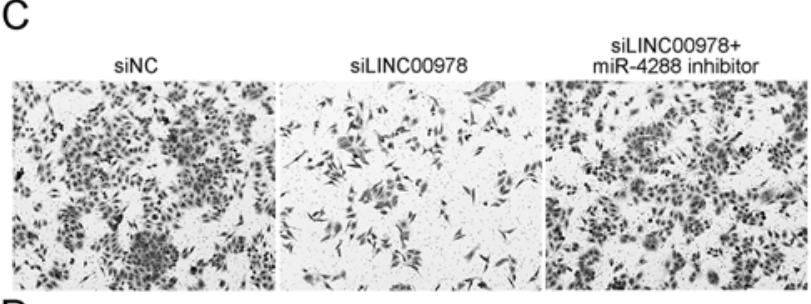

D

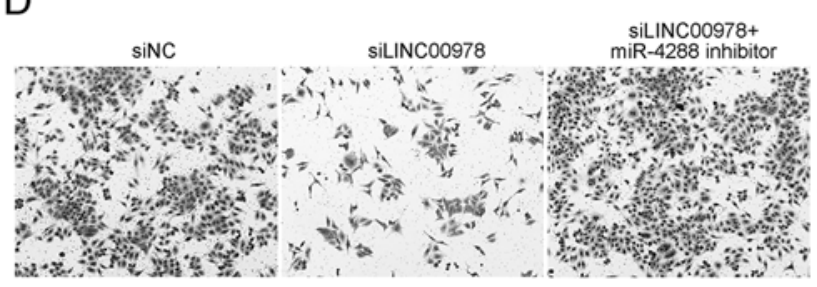

B
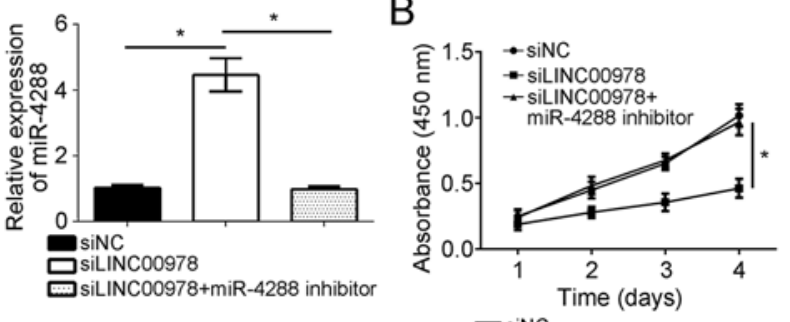

$\square^{\text {sinCINC00978 }}$

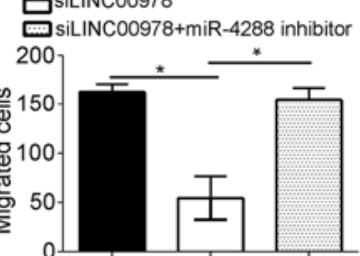

sinc

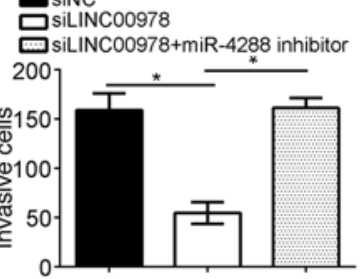

Figure 5. Inhibition of miR-4288 reverses the effects of LINC00978 knockdown on bladder cancer cells. (A) Relative expression of miR-4288 in transfected T2 4 cells by reverse transcription-quantitative polymerase chain reaction. (B) Cell Counting kit- 8 assay was used to determine cell proliferation of T24 cells. (C) Transwell and (D) Matrigel assays were used to detect cell migration and invasion of T24 cells, respectively (magnification, $\mathrm{x} 100$ ). * $<<0.05$. LINC00978, long intergenic non-protein coding RNA 978; miR-4288, microRNA-4288; si, small interfering; NC, negative control.

development and progression remains poorly understood. In the present study, the function of LINC00978 in BCa progression was investigated. The present study demonstrated that the LINC00978 expression level was significantly upregulated in BCa tissues and cell lines compared with adjacent normal tissues and a normal cell line, respectively. Knockdown of LINC00978 led to decreased proliferation of BCa cells in vitro, and overexpression of LINC00978 led to the opposite effect. Additionally, it was demonstrated that LINC00978 knockdown suppressed the migratory and invasive potential of BCa cells. The present study demonstrated a novel function of LINC00978 in BCa. Collectively, the present findings suggested that LINC00978 serves an oncogenic role in $\mathrm{BCa}$ and may represent a potential therapeutic target for the treatment of $\mathrm{BCa}$.

A previous study suggests that lncRNAs may serve as ceRNAs to sponge miRs and regulate gene expression (19). For example, small nucleolar RNA host gene 16 contributed to the tumorigenesis of cervical cancer cells by sponging 
miR-216-5p, which increased the expression level of zinc finger E-box binding homeobox 1 (20). lncRNA urothelial cancer associated 1 promoted proliferation and conferred cisplatin resistance in oral squamous cell carcinoma by suppressing miR-184 (21). IncRNA-ATB promoted epithelial-mesenchymal transition in a model of silica-induced pulmonary fibrosis by binding to miR-200c (22). X inactive specific transcript promoted gastric cancer progression by targeting miR-185, upregulating transforming growth factor $\beta 1$ expression level (23). A previous study identified that LINC00978 may serve as an oncogene in gastric and breast cancer (14,15). Furthermore, LINC00978 upregulation was associated with poor prognosis in gastric and breast cancer $(14,15)$. However, the mechanism underlying LINC00978 regulation of cancer progression remains unknown.

In the present study, to examine the mechanism of LINC00978, a bioinformatics analysis was performed. The present results suggested that miR-4288 may represent a target of LINC00978. A luciferase reporter assay suggested a direct interaction between LINC00978 and miR-4288 in BCa cells. Furthermore, it was identified that overexpression of miR-4288 inhibited LINC00978 expression in BCa cells, whereas, knockdown of LINC00978 promoted miR-4288 expression. The expression of LINC00978 was inversely correlated with miR-4288 in BCa tissues. Collectively, the present data suggested that LINC00978 may serve as a ceRNA of miR-4288. To investigate whether LINC00978 serves as an oncogene by sponging miR-4288, a rescue assay was performed. The present results suggested that miR-4288 inhibition reversed the effects of LINC00978 knockdown on $\mathrm{BCa}$ cell proliferation, migration and invasion. Therefore, LINC00978 contributed to BCa progression in vitro by inhibiting miR-4288.

A previous study suggests that miRNAs are able to regulate gene expression and function, by serving as tumor suppressors or oncogenes (24). Although the present data suggested that miR-4288 served as a tumor suppressor in $\mathrm{BCa}$, the function of miR-4288 has not been previously investigated, to the best of the authors' knowledge, and additional studies are required to understand the signaling pathways downstream of miR-4288 in BCa. Collectively, the present study investigated the role of LINC00978 in BCa progression and suggested that the LINC00978/miR-4288 axis may represent a potential therapeutic target for treating $\mathrm{BCa}$.

\section{Acknowledgements}

Not applicable.

\section{Funding}

The present study was supported by The Funds of Science Technology Department of Zhejiang Province (grant no. LGF18H160027; China).

\section{Availability of data and materials}

All data generated or analyzed during this study are included in this published article.

\section{Authors' contributions}

WW contributed to the conception and design of the present study. In addition, WW analyzed and interpreted the results, and wrote the manuscript. ZX, JW and RC performed the experiments. All authors read and approved the final manuscript.

\section{Ethics approval and consent to participate}

The present study was approved by The Ethics Committee of Tiantai People's Hospital of Zhejiang Province (approval no. 2016020342; Taizhou, China). Written informed consent was obtained from all enrolled patients.

\section{Patient consent for publication}

All patients within the present study provided consent for the publication of their data.

\section{Competing interests}

The authors declare that they have no competing interests.

\section{References}

1. Jemal A, Bray F, Center MM, Ferlay J, Ward E and Forman D: Global cancer statistics. CA Cancer J Clin 61: 69-90, 2011.

2. Ferlay J, Soerjomataram I, Dikshit R, Eser S, Mathers C, Rebelo M, Parkin DM, Forman D and Bray F: Cancer incidence and mortality worldwide: Sources, methods and major patterns in GLOBOCAN 2012. Int J Cancer 136: E359-E386, 2015.

3. Wang M, Guo C, Wang L, Luo G, Huang C, Li Y, Liu D, Zeng F, Jiang $\mathrm{G}$ and Xiao $\mathrm{X}$ : Long noncoding RNA GAS5 promotes bladder cancer cells apoptosis through inhibiting EZH2 transcription. Cell Death Dis 9: 238, 2018.

4. Tran MN, Goodwin Jinesh G, McConkey DJ and Kamat AM: Bladder cancer stem cells. Curr Stem Cell Res Ther 5: 387-395, 2010.

5. Feng $\mathrm{Z}$ and Wang B: Long non-coding RNA HNF1A-AS1 promotes cell viability and migration in human bladder cancer. Oncol Lett 15: 4535-4540, 2018.

6. Liao Z, Zhao J and Yang Y: Downregulation of lncRNA H19 inhibits the migration and invasion of melanoma cells by inactivating the NF- $\mathrm{KB}$ and PI3K/Akt signaling pathways. Mol Med Rep 17: 7313-7318, 2018.

7. Liu B, Ye B, Yang L, Zhu X, Huang G, Zhu P, Du Y, Wu J, Qin X, Chen $\mathrm{R}$, et al: Long noncoding RNA $\operatorname{lncKdm} 2 \mathrm{~b}$ is required for ILC3 maintenance by initiation of Zfp292 expression. Nat Immunol 18: 499-508, 2017.

8. Ye B, Liu B, Yang L, Zhu X, Zhang D, Wu W, Zhu P, Wang Y, Wang S, Xia P, et al: LncKdm2b controls self-renewal of embryonic stem cells via activating expression of transcription factor Zbtb3. EMBO J 37: pii: e97174, 2018.

9. Zhao R, Wang X, Wang H, Yu T, Wang Q, Yang X and Sun J: Inhibition of long noncoding RNA BDNF-AS rescues cell death and apoptosis in hypoxia/reoxygenation damaged murine cardiomyocyte. Biochimie 138: 43-49, 2017.

10. Zheng R, Lin S, Guan L, Yuan H, Liu K, Liu C, Ye W, Liao Y, Jia J and Zhang R: Long non-coding RNA XIST inhibited breast cancer cell growth, migration, and invasion via miR-155/CDX1 axis. Biochem Biophys Res Commun 498: 1002-1008, 2018.

11. He W, Zhong G, Jiang N, Wang B, Fan X, Chen C, Chen X, Huang $\mathrm{J}$ and Lin T: Long noncoding RNA BLACAT2 promotes bladder cancer-associated lymphangiogenesis and lymphatic metastasis. J Clin Invest 128: 861-875, 2018.

12. Liao X, Chen J, Liu Y, He A, Wu J, Cheng J, Zhang X, Lv Z, Wang $\mathrm{F}$ and Mei $\mathrm{H}$ : Knockdown of long noncoding RNA FGFR3- AS1 induces cell proliferation inhibition, apoptosis and motility reduction in bladder cancer. Cancer Biomark 21: 277-285, 2018. 
13. Luo J, Chen J, Li H, Yang Y, Yun H, Yang S and Mao X: LncRNA UCA1 promotes the invasion and EMT of bladder cancer cells by regulating the miR-143/HMGB1 pathway. Oncol Lett 14: 5556-5562, 2017

14. Fu M, Huang Z, Zang X, Pan L, Liang W, Chen J, Qian H, $\mathrm{Xu}$ W, Jiang P and Zhang X: Long noncoding RNA LINC00978 promotes cancer growth and acts as a diagnostic biomarker in gastric cancer. Cell Prolif 51, 2018.

15. Deng LL, Chi YY, Liu L, Huang NS, Wang L and Wu J: LINC00978 predicts poor prognosis in breast cancer patients. Sci Rep 6: 37936, 2016.

16. Livak KJ and Schmittgen TD: Analysis of relative gene expression data using real-time quantitative PCR and the 2(T)(-Delta DeltaC) method. Methods 25: 402-408, 2001.

17. Ploeg M, Aben KK and Kiemeney LA: The present and future burden of urinary bladder cancer in the world. World J Urol 27: 289-293, 2009.

18. Millan-Rodriguez F, Chechile-Toniolo G, Salvador-Bayarri J, Palou J, Algaba F and Vicente-Rodriguez J: Primary superficial bladder cancer risk groups according to progression, mortality and recurrence. J Urol 164: 680-684, 2000.

19. Li HJ, Sun XM, Li ZK, Yin QW, Pang H, Pan JJ, Li X and Chen W: LncRNA UCA1 promotes mitochondrial function of bladder cancer via the MiR-195/ARL2 signaling pathway. Cell Physiol Biochem 43: 2548-2561, 2017.
20. Zhu H, Zeng Y, Zhou CC and Ye W: SNHG16/miR-216-5p/ZEB1 signal pathway contributes to the tumorigenesis of cervical cancer cells. Arch Biochem Biophys 637: 1-8, 2018.

21. Fang Z, Zhao J, Xie W, Sun Q, Wang H and Qiao B: LncRNA UCA 1 promotes proliferation and cisplatin resistance of oral squamous cell carcinoma by sunppressing miR-184 expression. Cancer Med 6: 2897-2908, 2017.

22. Liu Y, Li Y, Xu Q, Yao W, Wu Q, Yuan J, Yan W, Xu T, Ji X and Ni C: Long non-coding RNA-ATB promotes EMT during silica-induced pulmonary fibrosis by competitively binding miR-200c. Biochim Biophys Acta Mol Basis Dis 1864: 420-431, 2018.

23. Zhang Q, Chen B, Liu P and Yang J: XIST promotes gastric cancer (GC) progression through TGF- $\beta 1$ via targeting miR-185. J Cell Biochem 119: 2787-2796, 2018.

24. TonouchiE, Gen Y,Muramatsu T, HiramotoH,Tanimoto K, Inoue J and Inazawa J: miR-3140 suppresses tumor cell growth by targeting BRD4 via its coding sequence and downregulates the BRD4-NUT fusion oncoprotein. Sci Rep 8: 4482, 2018. 